\title{
Precise Temperature Measurements and Earthquake Heat Associated with the 1999 Chi-Chi, Taiwan Earthquake
}

\author{
by Yasuyuki Kano, Jim Mori, Ryo Fujio, Takashi Yanagidani, Setsuro Nakao, \\ Hisao Ito, Osamu Matsubayashi, and Kuo-Fong Ma
}

\section{Introduction}

The frictional heat generated during earthquake faulting is thought to be the largest part ( $80 \%$ to $90 \%)$ of the total seismic energy budget, and geophysicists have long discussed the level of heat that should be observable (Brune et al., 1969; Lachenbruch and Sass, 1980; Scholz, 2002; Terada, 1930). Precise temperature measurements across the fault immediately after an earthquake can provide the most unambiguous answer; however, there has never been a significant near-fault temperature change observed for any previous large earthquake that can be attributed to the frictional heating. This is because there has been no appropriate site for temperature measurements around a fault at depth just after an earthquake. The most promising way to reach the fault zone in order to observe the frictional heat is to drill a borehole to the area where large slip occurs. There have been several drilling projects to reach deep areas of the fault zone, such as the Taiwan Chelungpu fault Drilling Project (TCDP), San Andreas Fault Observatory at Depth (SAFOD), and the planned NanTroSEIZE project. We reported the first successful temperature measurement of deep fault zone boreholes that was drilled by TCDP at the Chelungpu fault, Taiwan (Kano et al., 2006). An observation of a temperature increase, and thus an estimate of the heat generated, provides information about the frictional strength during faulting and the level of driving stress for an earthquake. These are key unknown values of important parameters that are necessary for understanding the physical process of earthquake ruptures.

In this paper we outline the results of the precise temperature measurement in TCDP Hole A as an attempt to directly measure the frictional heat produced by an earthquake.

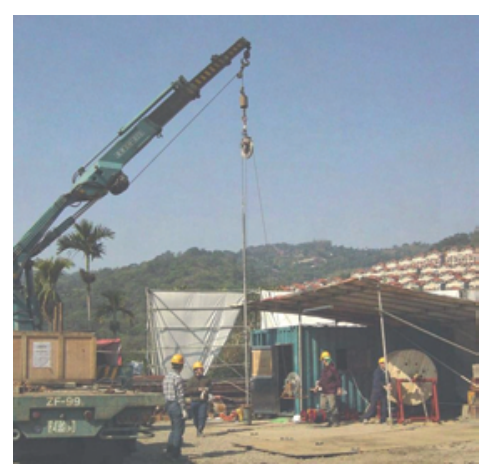

Figure 1. The precise temperature instrument that was installed in the well head of TCDP Hole A. cise temperature measurement. The details of our temperaturemeasurementin the Chelungpufaultanditsinterpretation are presented in Kano et al. (2006).

\section{Precise Measurements at TCDP Hole A}

Following the 21 September 1999 Chi-Chi earthquake, the TCDP bored two holes which penetrated the fault at depths of about $1100 \mathrm{~m}$ (Ma et al., 2006) near the town of DaKeng in the northern part of the rupture zone. During the earthquake, this area had large surface rupture, and a fault displacement of about $8 \mathrm{~m}$ is estimated from seismic data. The boreholes provided the rare opportunity to make temperature measurements in a fault zone with large slip from a recent earthquake. The precise temperature observations were carried out in one of the boreholes (Hole A) during September 2005, six years following the earthquake. The borehole is cased with steel pipe so that there is no water flow between the borehole and surrounding rock, enabling much more stable temperature measurements.

In order to obtain a high-resolution $\left(0.003^{\circ} \mathrm{C}\right)$ temperature profile, we developed a borehole instrument containing two quartz oscillator thermometers, separated by $3 \mathrm{~m}$. The instrument was slowly lowered (about $1.0 \mathrm{~m} \mathrm{~min}^{-1}$ ) and raised (about $0.4 \mathrm{~m} \mathrm{~min}^{-1}$ ) in the borehole between the depths of $900 \mathrm{~m}$ and $1250 \mathrm{~m}$, producing four independent temperature profiles across the fault zone, during 20 and 21 September (Fig. 1). The continuous recording of temperature at $10 \mathrm{~s}$ intervals produced $5-15$ readings per meter.

All the temperature profiles show small temperature signatures, which are a small temperature increase above the geothermal gradient in the region of the fault zone at the depth of $1111 \mathrm{~m}$. Kano et al. (2006) simply modeled this temperature signature as a residual temperature anomaly originating in frictional heat produced by fault slip.

\section{Modeling of Temperature Anomaly}

The temperature signature produced by frictional heat is modeled by heat conduction from the fault plane as a heat source. Here we simply modeled the signature assuming one-dimensional heat conduction, in which heat produced on a thin plane is conducted to the direction perpendicular to the plane (Officer, 1974). We assumed that all the heat generated was consumed to increase the temperature of thin 
fault surface. The temperature change at the point that $x \mathrm{~m}$ away from the fault surface after $t \mathrm{~s}$ is

$$
T(x, t)=\frac{S}{2 \sqrt{\pi \alpha t}} e^{-x^{2} / 4 \alpha t}
$$

where $\alpha$ is the heat diffusivity of the media surrounding the fault, and $S$ is the strength of the heat source, which is heat (product of shear stress, $\tau$, and fault slip, $u$ ) divided by specific heat, $c$, and the density, $\rho$, of the medium with units measured in $\mathrm{K} \mathrm{m}$ :

$$
S=\frac{\tau \cdot u}{c \cdot \rho}
$$

Figure $2 \mathrm{~A}$ shows the spatial variation of temperature signature after five years and ten years from the earthquake (calculated for the parameters $u=6 \mathrm{~m}, \tau=1.1 \mathrm{MPa}$, $c=1140 \mathrm{~J} \mathrm{~kg}^{-1} \mathrm{~K}^{-1}, \rho=2500 \mathrm{~kg} \mathrm{~m}^{-3}$, and $\left.\alpha=3.4 \times 10-7 \mathrm{~m}^{2} \mathrm{~s}^{-1}\right)$. The amplitude becomes smaller with an increase of $t$, and the shape of the signature becomes broader. The amplitude of the peak of the temperature anomaly is only $0.06 \mathrm{~K}$ after five years. Figure $2 \mathrm{~B}$ shows the temporal variation of temperature signature on the fault and at the point $1 \mathrm{~m}$ and $10 \mathrm{~m}$ apart from the fault plane.

\section{Spatial Variation of Thermal Conductivity}

The temperature profile is strongly affected by the variations of thermal conductivity of the surrounding material. Kano et al. (2006) interpreted the observed temperature profile assuming the background temperature gradient is constant. They ignored the effect of spatial variation of thermal conductivity on the temperature gradient; however, the spatial variation of thermal conductivity around the Chelungpu fault is large enough to affect the interpretation of the temperature anomaly (Matsubayashi et al., 2005). Using thermal conductivity data in Hole A, Tanaka et al. (2007) reinterpreted the temperature data by Kano et al. (2006) and suggested that the temperature signature detected by Kano et al. (2006) might be a result of thermal conductivity fluctuations, rather than a residual heat from frictional faulting. Tanaka et al. (2007) measured the thermal conductivity with core soaked in epoxy resin to fill the cracks. Doing this introduces error in the measurement of thermal conductivity because of the low value of thermal conductivity of epoxy resin compared to rocks. The effect of epoxy resin is not well evaluated. Their data, in addition, is not sampled around fault zone, which brings error to the prediction of temperature variation. To extract the heat signature produced by fault slip, we had to make

measurements of the spatial variation of material thermal conductivity and examine its effect.

The relationship between temperature gradient $(d T / d z)$ and thermal conductivity, $\kappa$, at depth, $z$, is

$$
\frac{d T}{d z}=\frac{q}{\kappa(z)}
$$

where $q$ is heat flow. Assuming constant $q$, we can predict a temperature variation produced only by the spatial variation of $\kappa$,

$$
T(z)=q \int \frac{1}{\kappa(z)} d z
$$

This means that the temperature gradient produced by constant heat flow is not constant in the medium that has spatial variation of thermal conductivity. Here we predict the background temperature gradient in the temperature profile from the thermal conductivity measured using the core samples of Hole B (Matsubayashi et al., 2005). They measured the thermal conductivity every $0.04-1.5 \mathrm{~m}$ for the cores in which water content is carefully preserved before the measurement. We assume that the thermal conductivity, and thus lithology and porosity, in Hole A and Hole B are continuous with 26-m depth difference. Figure 3 shows the predicted temperature profile assuming a constant heat flow of $60 \mathrm{~mW} \mathrm{~m}^{-2}$ together with the observed temperature anomaly. The depth of Hole B is shifted by $26 \mathrm{~m}$, which is the difference between the $1111 \mathrm{~m}$ fault zone detected in Hole A and the $1137 \mathrm{~m}$ fault zone in Hole B. To calibrate the response delay included in the observed temperature anomaly that is caused by thermal inertia of the temperature instrument, observed and predicted temperature anomalies are low-pass filtered $(40 \mathrm{~m})$. We can see peaks in the
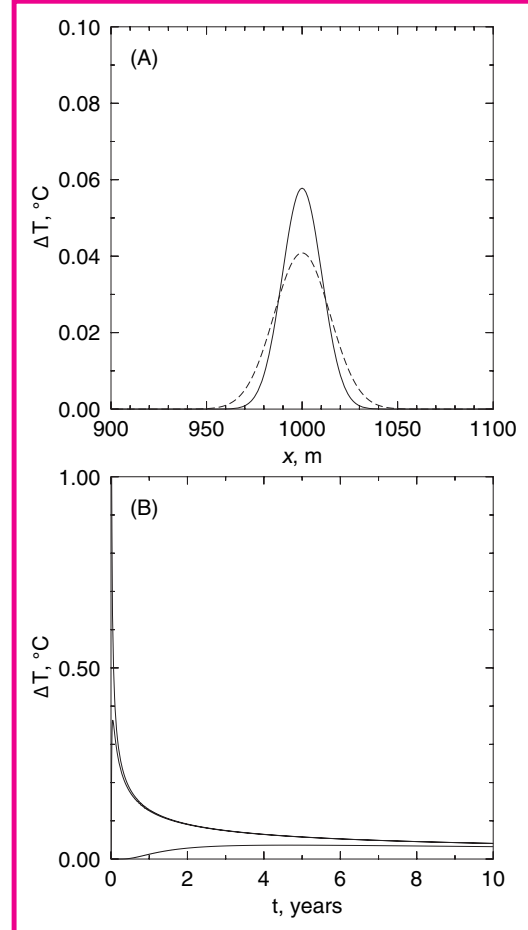

Figure 2. $[A]$ Spatial and $[B]$ temporal variations of temperature signature calculated for $u=6 \mathrm{~m}$. predicted temperature anomaly $(1110 \mathrm{~m}$, $1150 \mathrm{~m}$, and $1190 \mathrm{~m}$ ) compared to the observed temperature anomaly $(1110 \mathrm{~m}$ and $1190 \mathrm{~m}$ ). Those peaks in the corrected temperature observations reflect variations of thermal conductivity that are caused by differences of rock type and porosity around the fault zone.

The peak in the predicted temperature profile is smaller than the observed temperature anomaly around $1111 \mathrm{~m}$, which is modeled as the heat signature of fault slip in Kano et al. (2006), and is considered to be a temperature increase superimposed on background temperature fluctuation. The contribution of residual frictional heat produced by the fault slip is then smaller than the peak modeled in Kano et al. (2006). Thus, their estimation of heat produced gives an upper bound of heat generated by fault slip. The apparent coefficient of friction is estimated to be $0.04-0.08$. Laboratory 
determinations of the static coefficient of friction are generally quite high, 0.6 to 0.7 (Byerlee, 1978) or 0.35 to 0.5 for shales (Morrow et al., 1992), and would produce much higher amounts of heat if these values are used for the dynamic coefficient of friction.

The assumption that the thermal conductivities in Hole A and Hole B are continuous is not necessarily true, since the lithology and thermal property may be three-dimensionally inhomogeneous. The best way to remove the background temperature profile is to use thermal conductivity data from Hole A to predict

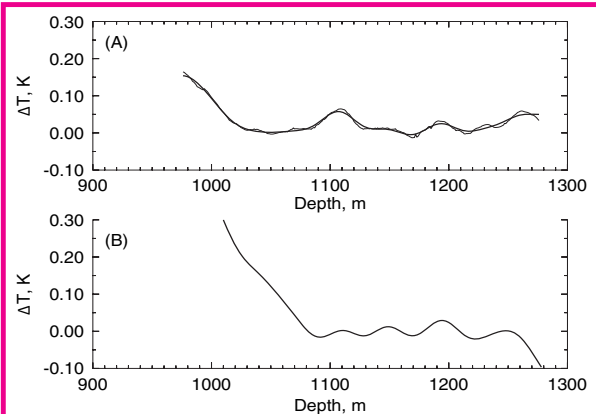

Figure 3. $[A]$ Observed temperature anomaly in Hole $A$ and $[B]$ temperature anomaly predicted from the thermal conductivity measurement of core from Hole $B$. The depth of Hole B is shifted by $26 \mathrm{~m}$, which is the difference between the $1111 \mathrm{~m}$ fault zone detected in Hole $\mathrm{A}$ and the $1137 \mathrm{~m}$ fault zone in Hole B. Thick lines shows the temperature anomalies that are low pass filtered with cutoff length of $40 \mathrm{~m}$ the temperature fluctuations contained in the temperature measurements from Hole A. There is, however, applicable thermal conductivity data measured only from the core of Hole B. To model the residual frictional heat more precisely, we need an appropriate depth correction between Hole A and Hole B.

\section{Summary}

Measuring temperature around the fault zones is a way to obtain knowledge of frictional heat produced during earthquakes and thus the energy budget, which are key unknown parameters that are necessary for understanding the physical process of earthquake ruptures. The spatial variations of material thermal conductivity may be another factor that affects the temperature signature, which is sometimes very similar to the temperature signature produced by the frictional heat of fault slip. To obtain the correct background temperature profile, we need to correct the observed temperature signature using thermal conductivities of the formation. When we make temperature measurement to seek residual frictional heat along the fault, it is important to measure material thermal properties that have enough resolution to remove the background temperature fluctuations. In our particular case, we do not have enough thermal conductivity data from Hole A to completely calibrate the temperature signature. The calculated heat in our present results was an upper bound, and it implies a very low level of dynamic friction during faulting for this region of large slip. The low level of friction we obtained needs to be confirmed for other events, and, if verified, indicates that low friction mechanisms are needed to explain the dynamic rupture process of large earthquakes.

\section{Acknowledgements}

This work was partially supported by the Kyoto University 21st Century Center of Excellence (KAGI21) and other Grants in Aid for Scientific Research (16253003) from the Ministry of Education, Culture, Sports, Science, and Technology of Japan and the National Science Council of Taiwan.

\section{References}

Brune, J., Henyey, T., and Roy, R., 1969. Heat flow, stress, and rate of slip along the San Andreas fault, California. J. Geophys. Res., 74:3821-3827.

Byerlee, J.D., 1978. Friction of rocks. Pure Appl. Geophys., 116:615-626, doi:10.1007/ BF00876528.

Kano, Y., Mori, J., Fujio, R., Ito., H., Yanagidani, T., Nakao, S., and Ma, K.-F., 2006. Heat signature on the Chelungpu Fault associated with the 1999 Chi-Chi, Taiwan earthquake. Geophys. Res. Lett., 33:L14306, doi:10.1029/ 2006GL026733.

Lachenbruch, A.H. and Sass, J.H., 1980. Heat flow and energetics of the San Andreas fault zone. J. Geophys. Res., 85:6185-6222.

Ma, K.-F., Song, S.-R., Tanaka, H., Wang, C.-Y., Hung, J.-H., Tsai, Y.-B., Mori, J., Song, Y.-F., Yeh, E.-C., Sone, H., Kuo, L.-W., and $\mathrm{Wu}$, H.Y., 2006. Slip zone and energetics of a large earthquake: Results from the Taiwan Chelungpu-fault Drilling Project (TCDP). Nature, 444:473-476, doi:10.1038.

Matsubayashi, O., Lin, W., Hirono, T., Song, S., and Hung, J., 2005. Characterization of unfractured wall rocks of TCDP Hole-B by combination of thermal-property and TDR measurements in laboratory. Eos Trans. AGU, 86(52), Fall Meet. Suppl., Abstract T51A-1321.

Morrow, C., Radney, B., Byerlee, J., 1992. Frictional strength and the effective pressure law of montmorillonite and illite clays. In Evans, B., and Wong, T.F. (Eds.), Fault Mechanics and Transport Properties of Rocks. San Diego, Calif. (Academic Press), 69-88.

Officer, C.B., 1974. Introduction to Theoretical Geophysics, New York (Springer-Verlag).

Scholz, C.H., 2002. The Mechanisms of Earthquake Faulting, 2nd Edition, New York (Cambridge University Press), 158-167.

Tanaka, H., Chen, W.M., Kawabata, K., and Urata, N., 2007. Thermal properties across the Chelungpu fault zone and evaluations of positive thermal anomaly on the slip zones: Are these residuals of heat from faulting? Geophys. Res. Lett., 34: L01309, doi:10.1029/2006GL028153.

Terada, T., 1930. On the heat generated by the deformation of the Earth crust. Bull. Earthquake Res. Inst., 8:377-383.

\section{Authors}

Yasuyuki Kano, Jim Mori, Ryo Fujio, Takashi Yanagidani, and Setsuro Nakao, Disaster Prevention Research Institute, Kyoto University, Gokasyo, Uji, 611-0011, Japan., e-mail: kano@rcep.dpri.kyoto-u.ac.jp.

Hisao Ito, Japan Agency for Marine Earth Science and Technology, 3173-25, Showa-machi, Kanazawa-ku, Yokohama, Kanazawa, 236-0001, Japan.

Osamu Matsubayashi, National Institute of Advanced Industrial Science and Technology, No.7, Higashi 1-1-1, Tsukuba, 305-8567, Japan.

Kuo-Fong Ma, National Central University, No.300, Jhongda Road, Jhongli City, Taoyuan County, 32001, Taiwan (R.O.C.). 